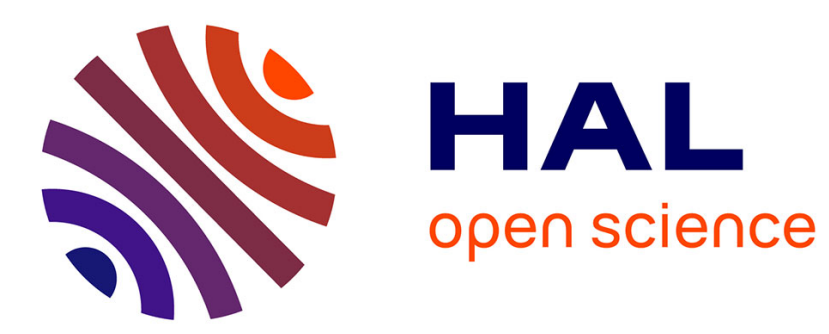

\title{
A Gradual Resource Allocation Technique for Massive MIMO-NOMA
}

\author{
Eric Pierre Simon, Joumana Farah, Pierre Laly, Gauthier Delbarre
}

\section{To cite this version:}

Eric Pierre Simon, Joumana Farah, Pierre Laly, Gauthier Delbarre. A Gradual Resource Allocation Technique for Massive MIMO-NOMA. IEEE Antennas and Wireless Propagation Letters, 2022, 21 (3), pp.476-480. 10.1109/LAWP.2021.3135981 . hal-03497334

\section{HAL Id: hal-03497334 https://hal.science/hal-03497334}

Submitted on 20 Dec 2021

HAL is a multi-disciplinary open access archive for the deposit and dissemination of scientific research documents, whether they are published or not. The documents may come from teaching and research institutions in France or abroad, or from public or private research centers.
L'archive ouverte pluridisciplinaire HAL, est destinée au dépôt et à la diffusion de documents scientifiques de niveau recherche, publiés ou non, émanant des établissements d'enseignement et de recherche français ou étrangers, des laboratoires publics ou privés. 


\title{
A Gradual Resource Allocation Technique for Massive MIMO-NOMA
}

\author{
Eric Pierre Simon ${ }^{1}$, Joumana Farah ${ }^{2}$, Pierre Laly ${ }^{1}$, Gauthier Delbarre ${ }^{1}$
}

\begin{abstract}
In this paper, a new perspective for channel allocation and user pairing is proposed for a crowded downlink system using massive multiple-input multiple-output (MIMO), non-orthogonal multiple access (NOMA), and Zero-forcing (ZF) beamforming. The proposed user clustering technique allocates users to subbands and beams by a gradual relaxation of the correlation constraints in such a way to enhance the conditioning of the channel matrix. Channel measurements are performed in this context to validate the proposed technique in an experimental setup. Results show an important gain in performance in terms of both throughput and fairness with respect to orthogonal multiple access and to two state-of-the-art NOMA clustering methods.
\end{abstract}

Index Terms-Non Orthogonal Multiple Access, Massive Multiple-Input Multiple-Output, Zero-Forcing Beamforming, Channel Allocation, Channel Mearsurements.

\section{INTRODUCTION}

Lately, massive Multiple Input Multiple Output (MIMO) systems have constituted a significant research stream for leveraging the saturating performance of $4 \mathrm{G}$ mobile networks [1]. However, their implementation has raised a plethora of questions. A major one is the number of users that can be served on a common time-frequency resource through beamforming. This issue was addressed in [2] where the authors recommend a ratio between the number of antennas and beams from 2 to 8 . In [3], the number of antennas required to spatially separate users was shown to be about 3 to 4 times the number of beams. With such usage limitations, it is believed that additional improvements must be brought to reap the maximum benefit from massive multi-antenna architectures. In this context, Non Orthogonal Multiple Access (NOMA) [4][9] is overseen as an efficient ally to massive MIMO. By an appropriate power multiplexing of two or more signals on the same time-frequency and/or spatial resource (beam), powerdomain NOMA can easily overtake the performance of its Orthogonal Multiple Access (OMA) counterpart.

Several studies have tackled the resource allocation and beamforming problems in massive MIMO-NOMA [10]-[15]. In [14], optimization of intra-beam power allocation is studied for a single-beam MIMO system with two users. In [12], linear beamforming and power allocation are optimized to maximize system capacity. In [10], a simple solution is proposed for the allocation of several users on a single subband by clustering highly channel-correlated users with maximum channel gain

E.P. Simon, P. Laly and G. Delbarre are with the University of Lille and IEMN lab, Villeneuve d'Ascq, France. E.P. Simon is also a member of IRCICA. e-mail: eric.simon@univ-lille.fr.

J. Farah is with the Department of Electricity and Electronics, Faculty of Engineering, Lebanese University, Roumieh, Lebanon. e-mail: joumanafarah@ul.edu.lb. difference. In [15], a location-based beamforming MIMONOMA system is studied to determine the best user multiplexing scheme among several ones, depending on users positions. Maximal-Ratio-Transmission (MRT) beamforming was preferred whenever possible because of its limited complexity. However, the conclusions are drawn based on simple simulated environments that do not take into account the complexity of a practical environment.

Little work has been done to validate the combination of NOMA clustering and beamforming in massive MIMO through practical channel measurements. In [16], the method of [10] is generalized to the context of multi-subband allocation while considering a variable number of clusters and practical channel sounding techniques. However, a moderate number of users is accommodated on the spatial dimensions, preventing any significant gain in NOMA, with respect to OMA, when Zero-Forcing (ZF) beamforming is used.

In this letter, we consider a crowded system with a high number of active users to be served on the available timefrequency and spatial resources. Such system can correspond to low-latency applications in crowded areas where mobile users or Internet of Things (IoT) devices are expected to receive variable amounts of data in short time periods. This data can be, for instance, an urgent update of the devices databases or software. For this purpose, we exploit the massive MIMO system resources to their full extent by loading as many users as possible on each available subband and profit from NOMA to enhance system performance, compared to OMA. Since MRT was shown in [16] to yield much worse performance than $\mathrm{ZF}$, this work focuses on $\mathrm{ZF}$ beamforming. We propose a novel gradual correlation-thresholding resource allocation technique that proves that NOMA is particularly interesting in crowded contexts thanks to a better conditioning of the channel matrices.

The letter is organized as follows: the system model is first described in Section II, followed by the channel measurement campaign in Section III. Section IV presents the proposed resource allocation technique. Results and conclusions are provided in Section V.

\section{MASSIVE MIMO-NOMA System}

The studied system consists of a single cell with a unique base station (BS) equipped with $M=64$ antennas. The BS serves a large number of $K$ randomly deployed users. Such users can be mobile cellular transceivers or IoT devices. The system bandwidth $B$ is divided into $S$ subbands, each one consisting of an integer number of OFDM (Orthogonal Frequency Division Multiplexing) subcarriers. The BS performs 
$\mathrm{ZF}$ beamforming separately on each subband $s$ with a number of beams $N_{s}$ over $s$. The system is expected to serve all users on the available bandwidth so that in case of OMA, $\sum_{s=1}^{S} N_{s}=K$. In case NOMA is used, two or more users, constituting a cluster, can be grouped within the same beam. In the current work, and as is usually done in most works in downlink NOMA [4] [9] [17], the maximum cluster size is set to 2 to limit the Successive Interference Cancellation (SIC) complexity at the user equipment. The signal transmitted by the BS on subband $s$ can be written as:

$$
\mathbf{x}_{s}=\sum_{n=1}^{N_{s}} \sqrt{P_{n, s}} \mathbf{w}_{n, s} x_{n, s}
$$

$\mathbf{W}_{n, s}$ is the $n^{\text {th }}$ column of the $M \times N_{s}$ precoding matrix $\mathbf{W}_{s}$ of the $s^{\text {th }}$ subband. $P_{n, s}$ is the power transmitted by the BS on the $n^{\text {th }}$ beam of subband $s$. Each beam can either accommodate a single user or a pair of users. In case the $n^{\text {th }}$ beam supports a cluster of two users, signal $x_{n, s}$ is expressed by $x_{n, s}=$ $\sqrt{\alpha_{n, s, 1}} m_{n, s, 1}+\sqrt{\alpha_{n, s, 2}} m_{n, s, 2}$, where $m_{n, s, 1}$ and $m_{n, s, 2}$ are the transmitted signals of the two paired users on beam $n$ of subband $s$, with $E\left[\left|m_{n, s, i}\right|^{2}\right]=1, \forall n, s, i$ and $\alpha_{n, s, 1}+$ $\alpha_{n, s, 2}=1, \forall n, s$. The corresponding received signal at the level of the $i^{\text {th }}$ receiver, $i=1,2$, is given by:

$$
y_{n, s, i}=\mathbf{h}_{n, s, i} \mathbf{x}_{s}+q_{n, s, i}
$$

where $\mathbf{h}_{n, s, i}$ is the $1 \times M$ channel vector of user $i$ on beam $n$ of subband $s . q_{n, s, i}$ is an additive white complex Gaussian noise with zero mean and constant variance $\sigma^{2}$. On each subband and each cluster, users are sorted by their channel gains such that $\left\|\mathbf{h}_{n, s, 1}\right\|>\left\|\mathbf{h}_{n, s, 2}\right\|$, and the user indexed by $i=1$ (resp. $i=2$ ) is denoted as the strong (resp. weak) user in the cluster. For NOMA clusters, ZF beamforming is performed based on the channel gains of the strong users, as was done in [10] and [16]. Consider the $N_{s} \times M$ channel matrix $\mathbf{H}_{s}$ constituted by the channel vectors of the strong users within clusters and those of unique users of single-user beams. The ZF precoding matrix is first calculated as $\tilde{\mathbf{W}}_{s}=\mathbf{H}_{s}^{H}\left(\mathbf{H}_{s} \mathbf{H}_{s}^{H}\right)^{-1}$. Then, each beam precoding vector is normalized as follows: $\mathbf{w}_{n, s}=$ $\frac{\tilde{\mathbf{w}}_{n, s}}{\left\|\tilde{\mathbf{w}}_{n, s}\right\|}$, where $\tilde{\mathbf{w}}_{n, s}$ is the $n^{\text {th }}$ column of $\tilde{\mathbf{W}}_{s}$.

Let $g_{n, s, i, k}=\mathbf{h}_{n, s, i} \mathbf{w}_{k, s}$ be the equivalent channel gains of the $i^{\text {th }}$ user on beam $n$ of subband $s$. When $k \neq n,\left|g_{n, s, i, k}\right|^{2}$, multiplied by $P_{k, s}$, quantifies the amount of interference caused by beam $k$ on beam $n$. Besides, $g_{n, s, i, n}$ is the useful channel gain of the $n^{\text {th }}$ beam. Inside each NOMA beam, the strong user can remove intra-cluster interference (ICI) by SIC to remove the signal of the weak user before proceeding with the decoding of its own signal. According to the theory behind NOMA [9], [18], the weak user on each beam does not perform SIC. Such user suffers from ICI and also from inter-beam interference (IBI) induced by users signals from other beams. Assuming perfect SIC at the level of strong users, the achieved rates by the strong and weak users of two-users beams are respectively given by:

$$
\mathrm{R}_{n, s, 1}=\log _{2}\left(1+\frac{\left|g_{n, s, 1, n}\right|^{2} P_{n, s} \alpha_{n, s, 1}}{\sum_{k=1, k \neq n}^{N_{s}}\left|g_{n, s, 1, k}\right|^{2} P_{k, s}+\sigma^{2}}\right),
$$

$$
\begin{aligned}
& \mathbf{R}_{n, s, 2}=\log _{2}(1+ \\
& \left.\frac{\left|g_{n, s, 2, n}\right|^{2} P_{n, s} \alpha_{n, s, 2}}{\left|g_{n, s, 2, n}\right|^{2} P_{n, s} \alpha_{n, s, 1}+\sum_{k=1, k \neq n}^{N_{s}}\left|g_{n, s, 2, k}\right|^{2} P_{k, s}+\sigma^{2}}\right) .
\end{aligned}
$$

The rate of a user in a single-user beam is:

$$
\mathrm{R}_{n, s}=\log _{2}\left(1+\frac{\left|g_{n, s, n}\right|^{2} P_{n, s}}{\sum_{k=1, k \neq n}^{N_{s}}\left|g_{n, s, k}\right|^{2} P_{k, s}+\sigma^{2}}\right),
$$

where $g_{n, s, k}=\mathbf{h}_{n, s} \mathbf{w}_{k, s}$, with $\mathbf{h}_{n, s}$ the channel vector of the single user assigned to beam $n$ on subband $s$. Note that with ZF beamforming, the IBI terms in (3) and (5) are cancelled.

\section{DESCRIPTION OF THE EXPERIMENTAL CHANNEL MEASUREMENTS}

Channel measurements are performed with the MaMIMOSA (massive MIMO sounder architecture) radio channel sounder of the universities of Lille and Ghent [19], [20]. At the transmitter, an $8 \times 8$ vertical planar patch antenna array is deployed. The receiver is equipped with a single EM-6116 omnidirectional antenna. MaMIMOSA operates at $5.89 \mathrm{GHz}$ with $80 \mathrm{MHz}$ bandwidth. Before transmission, data are modulated using OFDM with 8192 subcarriers and a subcarrier spacing of $12.21 \mathrm{kHz}$. The streamshot mode of MaMIMOSA described in [20] is used for the measurements campaign: the subcarriers are uniformly distributed on a subset of 8 antennas, yielding a frequency spacing between two consecutive measured subcarriers on a single antenna of 97.66 $\mathrm{kHz}$. Hence, 8 OFDM symbols are necessary to measure the whole channel matrix between the transmitter and the receiver.

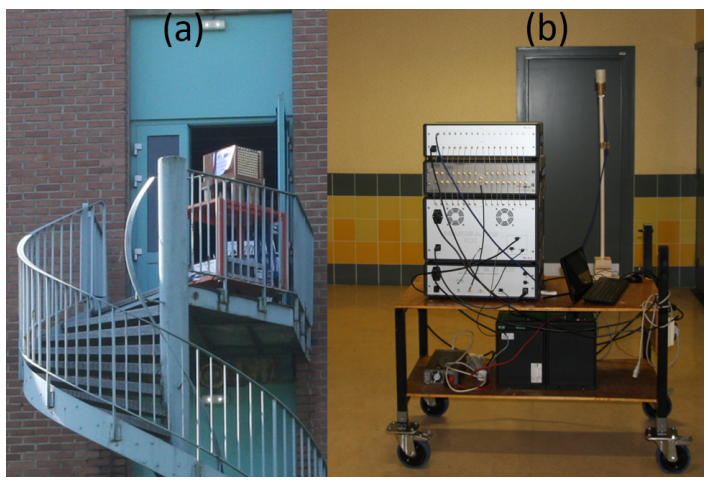

Fig. 1: (a) Tx, (b) $\mathrm{Rx}$

The transmitter (Tx) is positioned on a $10 \mathrm{~m}$ high platform on the P4 building of the Campus of the University of Lille, Fig. 1(a). The receiver ( $\mathrm{Rx})$ is deployed at a large number (650) of outdoor and indoor positions. The indoor environment corresponds to the interior of 3 floors of the P3 building and the ground floor of the P2 and P7 buildings-the interior consists of office and laboratory rooms. Outdoor positions are randomly taken within a radius of $300 \mathrm{~m}$ with respect to Tx. These configurations were chosen to emulate a crowded cell of a mobile system. The exact $\mathrm{Tx}$ and $\mathrm{Rx}$ positions on the campus and building maps can be found in [21]. 


\section{PROPOSED CHANNEL ALLOCATION AND PAIRING TECHNIQUE}

In this section, we describe the proposed resource allocation technique for the OMA and NOMA settings. The description will tackle the case of a fully-loaded system, where $K=M S$. However, the algorithm could also be applied for simpler use cases where $K<M S$. Let $\mathbf{h}_{k, s}, k=1, \ldots, K, s=1, \ldots, S$, the $1 \times M$ channel vector of user $k$ measured on the $s$ th subband. Note that, at this point, the beam index $n$ has been discarded in the notation and replaced by the user index $k$ since the assignment of users to beams has not been performed yet. In the proposed technique, users are greedily assigned to subbands using a gradual relaxation of the correlation constraints on beams. The channel correlation is used to assess the degree of orthogonality between the channel vectors of two users $k$ and $j$ on a subband $s$ and is given by:

$$
\rho_{k, j, s}=\frac{\left|\mathbf{h}_{k, s} \mathbf{h}_{j, s}^{H}\right|}{\left\|\mathbf{h}_{k, s}\right\|\left\|\mathbf{h}_{j, s}\right\|} .
$$

The allocation starts with an OMA phase developed in Algorithm 1, with an initial value of a correlation threshold denoted by $\beta$. Note that Matlab notation is used for loops, matrices, and vector indices. The algorithm starts by sorting the users priorities based on the channel gain difference between their best subband and their next best subband (steps 1 to 5), i.e., the user $k$ to be assigned first is the one for which the second-best channel state is much worse than the best one. $k$ is assigned its best subband $s$, unless $s$ has already $M$ assigned users, or if there exists at least an assigned user $j$ to $s$ s.t. $\rho_{k, j, s}>\beta$. In such a case, the next best subband is tested for $k$, and so on. The first iteration of the algorithm attempts the assignment of all users by priority order. Those that cannot be assigned will be attempted at the next iteration with a value of $\beta$ increased by a step $\Delta \beta$, and the process is repeated until all users have been assigned to subbands.

The second phase of the allocation technique deals with the NOMA pairing. Let $\mathbf{u}$ be a vector containing a concatenation of the last $M / 2$ users that were allocated on each subband in the OMA phase. Such users correspond to those with the worst orthogonality conditions since they were assigned at the later stages of the gradual relaxation procedure. The aim of Algorithm 2 is to reallocate this set of users by pairing them on appropriate beams and subbands while keeping the first $S M / 2$ allocated users on the beams assigned to them by Algorithm 1. Two correlation thresholds are now used, namely $\beta_{1}$ for ensuring a certain orthogonality level between users in different beams, and $\beta_{2}$ for ensuring a certain correlation level between paired users on the same beam. The maximum value of $\beta_{1}$ is always ensured to be no higher than the minimum value of $\beta_{2}$. Using the gradual relaxation of the two correlation thresholds, the set of possible pairs $\mathcal{P}$ with their corresponding subbands is constituted (steps 1 to 17). It is sorted by the decreasing order of correlation constraints, i.e., the paired users of the first pair have the strongest cross-correlation while having the highest level of orthogonality with other beams users. In steps 18 to 22 , the users are assigned to beams, by correlation order, as strong or weak users depending on their respective channel gain order.
At the end, users that could not be paired with others are reallocated to their original subband from the OMA phase. After OMA and NOMA allocations, ZF beamforming is performed as explained in Section II. Note that in the case where a nonfully-loaded system is used, i.e., $K<M S$, the initialization of the matrix $\mathbf{A}_{\text {NOMA }}^{(1)}$ will be done with an adaptive size instead of the $M / 2$ used in the current description.

In this work, an equal inter-beam and intra-beam power distribution of the BS power budget was adopted. More elaborate power allocation techniques [16] [9] [22] [17] were proposed in previous works that could be included within the proposed allocation strategies. However, they are beyond the scope of this paper, where we focus on proposing an enhanced strategy for channel allocation and user pairing.

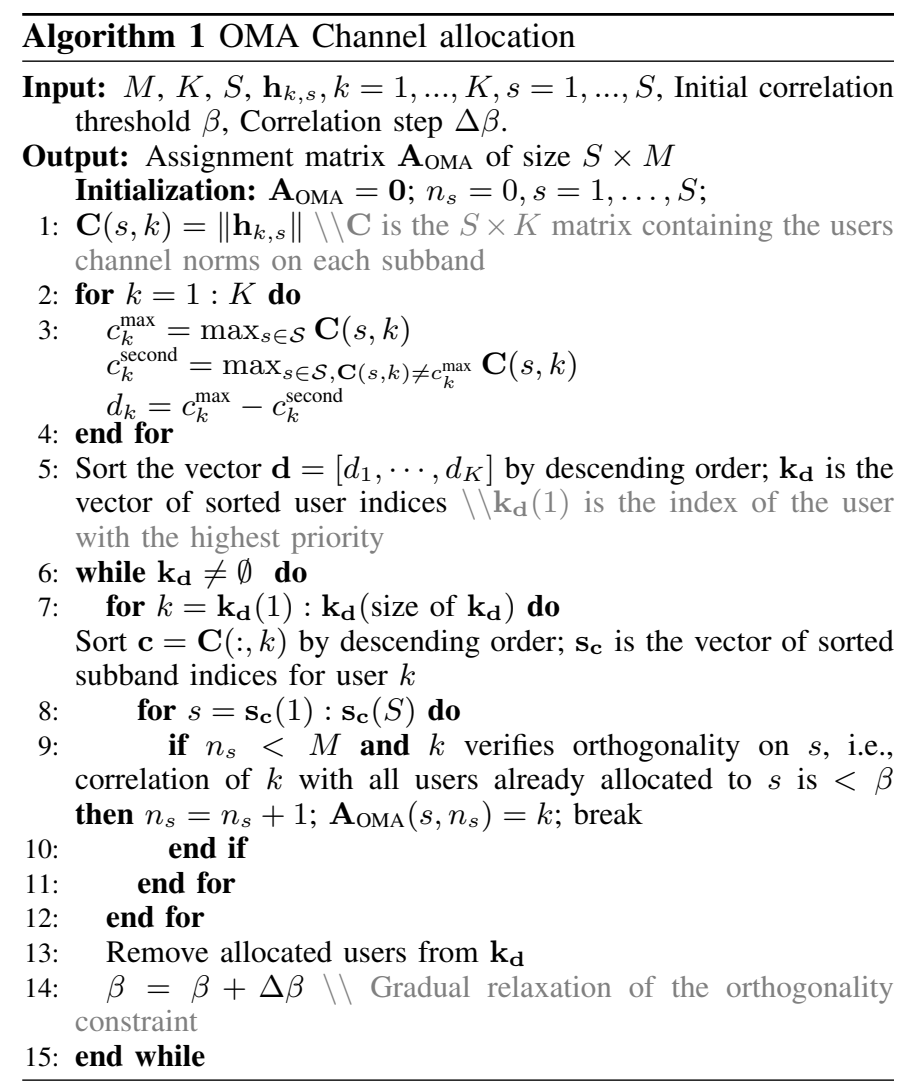

\section{RESUlTS AND CONCLUSION}

The performance of the proposed technique is assessed over a large number of system configurations (1000), each one with a subset of $K=320$ users positions randomly chosen from the set of 650 measured positions. $S=5$ subbands are considered, each one with a bandwidth of $195.32 \mathrm{kHz}$ (equivalent to 2 measured subcarriers), which is close to the $5 \mathrm{G}$ resource block bandwidth of $180 \mathrm{kHz} . M=64$ antennas are considered. These parameters correspond to a full-load usage of spatial resources in OMA $(K=S M)$. The following algorithm parameters were considered: initial value of $\beta=0.1$ in OMA, $\Delta \beta=\Delta \beta_{1}=\Delta \beta_{2}=0.1, \beta_{1 \text { (init) }}=0.1, \beta_{2 \text { (init) }}=0.9$, $\beta_{2(\min )}=0.3$.

Our proposed technique is compared to those presented in [10] and [12]. Fig. 2 shows the average of the total 

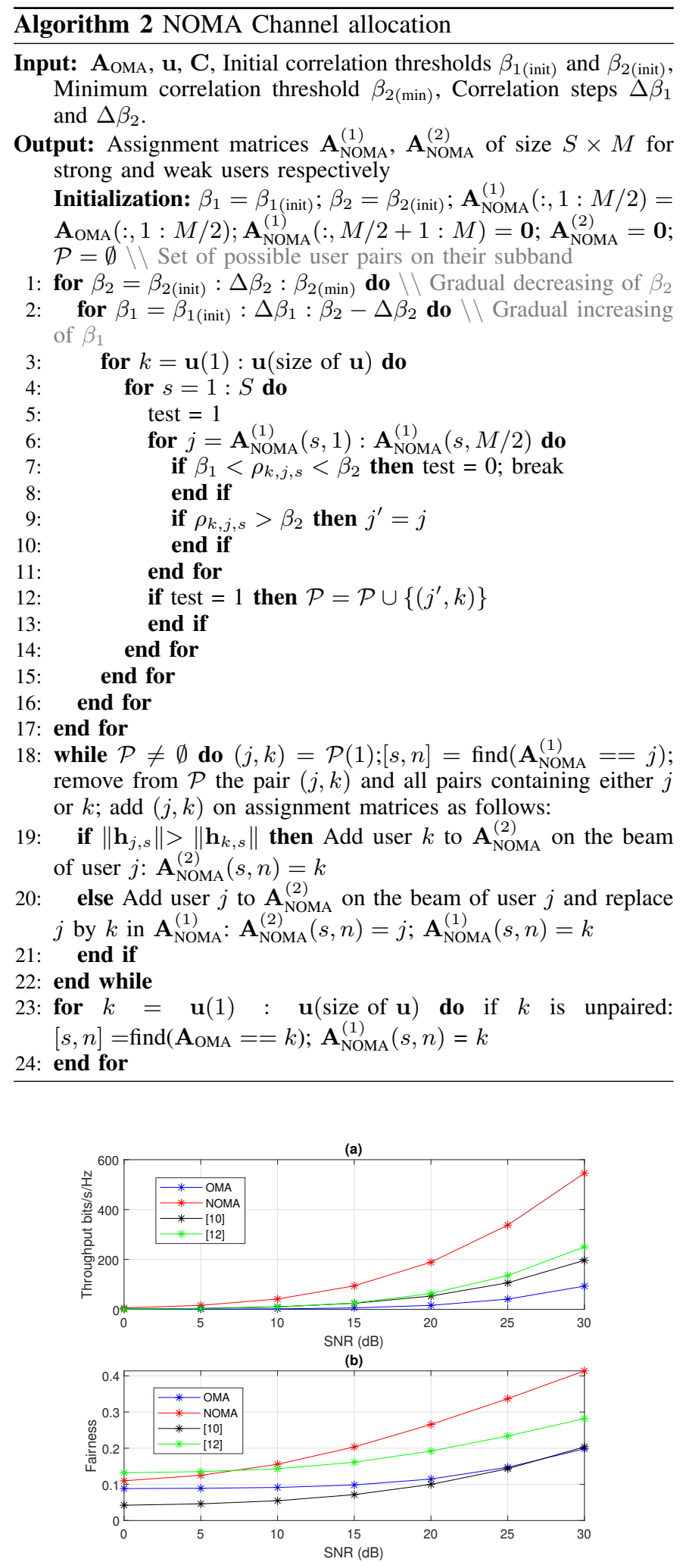

Fig. 2: Throughput (a) and Jain Fairness (b) vs. the SNR

throughput and Jain fairness index [23] as a function of the Signal-to-Noise-Ratio (SNR). The throughput is defined as $\sum_{s=1}^{S} \sum_{n=1}^{N_{s}} \sum_{i=1}^{2} \mathrm{R}_{n, s, i}$ and $\sum_{s=1}^{S} \sum_{n=1}^{N_{s}} \mathrm{R}_{n, s}$ for NOMA and OMA respectively, where $\mathrm{R}_{n, s, i}, i=1,2$, and $\mathrm{R}_{n, s}$ are given by eqts (3), (4) and (5). As for the Jain fairness index,

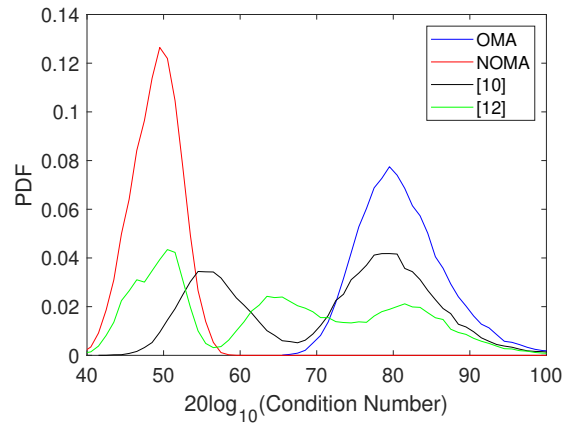

Fig. 3: PDF of the condition number of the channel matrix

it is given by: $\left.J=\left(\sum_{k=1}^{K} R_{k}\right)^{2} /\left(K \cdot \sum_{k=1}^{K} R_{k}^{2}\right)\right)$, where $R_{k}$ is the achieved rate by user $k$, given by either $\mathrm{R}_{n, s, i}, i=1,2$, or $\mathrm{R}_{n, s}$. The results show the superiority of our proposed NOMA technique compared to OMA as well to [10] and [12]. The throughput gain of NOMA with respect to [10] and [12] quickly increases with the SNR and reaches 353 and 296 bits/s/Hz respectively at SNR $=30 \mathrm{~dB}$. Furthermore, our allocation technique achieves a higher degree of fairness between users, compared to all other methods.

In Fig. 3, the probability density function (PDF) of the condition number $(\mathrm{CN})$ [3] of matrix $\mathbf{H}_{s}$ is represented for the four methods. The average $\mathrm{CN}$ is resp. 49, 81, 71 and $69 \mathrm{~dB}$ for NOMA, OMA, [10] and [12]. This clearly shows that our NOMA technique allows the best conditioning of the channel matrix among the four methods, which greatly influences system performance. Indeed, by gradually assigning users to beams, the NOMA method significantly reduces the number of beams per subband, which is 34 on average for NOMA, compared to 64, 58 and 56 for OMA, [10] and [12], respectively. The value of 34 corresponds to a ratio around 2 between the number of beams and that of transmit antennas and is in accordance with the recommendations of [2].

On the other hand, these observations are contrary to those of the previous work in [3], where the angle-to-interference factor was shown to be the most representative metric of system performance. In our work with a full-load usage of the spatial dimensions in OMA, such factor is always close to 1 , which is in accordance with the results of Fig. 9 in [3]. Therefore, the $\mathrm{CN}$ is the most representative metric in the fullload context. Moreover, the gain obtained by NOMA can also be explained by the fact that, due to the Cauchy-Schwartz inequality, the fewer rows of the channel matrix $\mathbf{H}_{s}$ that a particular $\tilde{\mathbf{w}}_{n, s}$ is constrained to be orthogonal to, the smaller its norm [24], and thus the larger the equivalent channel gains $g_{n, s, 1, n}$, which is favorable to throughput enhancement.

In conclusion, a novel gradual channel allocation technique was proposed in the context of a crowded wireless system based on massive MIMO and NOMA. By gradually relaxing the correlation constraints for user assignment to subbands, an important gain in throughput and fairness was achieved with respect to OMA and two reference NOMA methods. Our technique allows a better conditioning of the channel matrix, which is reflected by the conditioning number that shows to be truly representative of the achieved performance. 


\section{REFERENCES}

[1] R. Chataut and R. Ak1, "Massive MIMO systems for 5G and beyond networks-overview, recent trends, challenges, and future research direction," Sensors, vol. 20, no. 10, p. 2753, 2020.

[2] E. Björnson, E. G. Larsson, and M. Debbah, "Massive MIMO for maximal spectral efficiency: How many users and pilots should be allocated?" IEEE Transactions on Wireless Communications, vol. 15, no. 2, pp. 1293-1308, 2016.

[3] J. Flordelis, F. Rusek, X. Gao, G. Dahman, O. Edfors, and F. Tufvesson, "Spatial separation of closely-located users in measured massive MIMO channels," IEEE Access, vol. 6, pp. 40 253-40266, 2018.

[4] A. Benjebbour, A. Li, Y. Saito, Y. Kishiyama, A. Harada, and T. Nakamura, "System-level performance of downlink NOMA for future LTE enhancements," in Globecom Workshops (GC Wkshps). IEEE, 2013, pp. 66-70.

[5] Y. Liu, Z. Qin, M. Elkashlan, Z. Ding, A. Nallanathan, and L. Hanzo, "Non orthogonal multiple access for 5G and beyond," Proceedings of the IEEE, vol. 105, no. 12, pp. 2347-2381, 2017.

[6] S. Özyurt, E. P. Simon, and J. Farah, "NOMA with zero-forcing VBLAST," IEEE Communications Letters, vol. 24, no. 9, pp. 2070-2074, 2020.

[7] J. Farah, E. P. Simon, P. Laly, and G. Delbarre, "Efficient combinations of NOMA With distributed antenna systems based on channel measurements for mitigating jamming attacks," IEEE Systems Journal, vol. 15, no. 2, pp. 2212-2221, 2021.

[8] J. Farah, J. Akiki, and E. P. Simon, "Energy-efficient techniques for combating the influence of reactive jamming using non-orthogonal multiple access and distributed antenna systems," in 2019 Wireless Telecommunications Symposium (WTS), 2019, pp. 1-7.

[9] J. Farah, A. Kilzi, C. A. Nour, and C. Douillard, "Power minimization in distributed antenna systems using non-orthogonal multiple access and mutual successive interference cancellation," IEEE Transactions on Vehicular Technology, vol. 67, no. 12, pp. 11 873-11 885, 2018.

[10] B. Kim, S. Lim, H. Kim, S. Suh, J. Kwun, S. Choi, C. Lee, S. Lee, and D. Hong, "Non-orthogonal multiple access in a downlink multiuser beamforming system," in Military Communications Conference, MILCOM. IEEE, 2013, pp. 1278-1283.

[11] Z. Ding, R. Schober, and H. V. Poor, "A general MIMO framework for NOMA downlink and uplink transmission based on signal alignment," IEEE Transactions on Wireless Communications, vol. 15, no. 6, pp. $4438-4454,2016$

[12] S. Ali, E. Hossain, and D. I. Kim, "Non-orthogonal multiple access (NOMA) for downlink multiuser MIMO systems: User clustering, beamforming, and power allocation," IEEE access, vol. 5, pp. 565-577, 2016.

[13] X. Chen, F.-K. Gong, G. Li, H. Zhang, and P. Song, "User pairing and pair scheduling in massive MIMO-NOMA systems," IEEE Communications Letters, vol. 22, no. 4, pp. 788-791, 2018.

[14] Z. Xiao, L. Zhu, J. Choi, P. Xia, and X.-G. Xia, "Joint power allocation and beamforming for non-orthogonal multiple access (NOMA) in 5G millimeter-wave communications," IEEE Transactions on Wireless Communications, vol. 17, no. 5, pp. 2961-2974, 2018.

[15] J. Wang, Y. Li, C. Ji, Q. Sun, S. Jin, and T. Q. S. Quek, "Locationbased MIMO-NOMA: multiple access regions and low-complexity user pairing," IEEE Transactions on Communications, vol. 68, no. 4, pp. 2293-2307, 2020.

[16] E. P. Simon, J. Farah, and P. Laly, "Performance evaluation of massive MIMO with beamforming and nonorthogonal multiple access based on practical channel measurements," IEEE Antennas and Wireless Propagation Letters, vol. 18, no. 6, pp. 1263-1267, 2019.

[17] M.-R. Hojeij, J. Farah, C. A. Nour, and C. Douillard, "Resource allocation in downlink non-orthogonal multiple access (NOMA) for future radio access," in Vehicular Technology Conference (VTC Spring), IEEE 81st. IEEE, 2015, pp. 1-6.

[18] Z. Ding, Z. Yang, P. Fan, and H. V. Poor, "On the Performance of NonOrthogonal Multiple Access in 5G Systems with Randomly Deployed Users," IEEE Signal Process. Lett., vol. 21, no. 12, pp. 1501-1505, Dec. 2014.

[19] P. Laly, D. P. Gaillot, G. Delbarre, M. Van den Bossche, G. Vermeeren, F. Challita, E. Tanghe, E. P. Simon, W. Joseph, L. Martens et al., "Massive radio channel sounder architecture for $5 \mathrm{G}$ mobility scenarios: MaMIMOSA," in 14th IEEE European Conference on Antennas and Propagation (EuCAP), 2020, pp. 1-5.

[20] D. Gaillot, P. Laly, N. Dahmouni, G. Delbarre, M. Van den Bossche, G. Vermeeren, E. Tanghe, E. Simon, W. Joseph, L. Martens et al., "Measurement of the V2I massive radio channel with the MaMIMOSA sounder in a suburban environment," in 2021 15th European Conference on Antennas and Propagation (EuCAP). IEEE, 2021, pp. 1-4.

[21] E. Simon, J. Farah, P. Laly, and G. Delbarre, "Report on the massive MIMO channel measurement campaign," University of Lille, Tech. Rep., 2021. [Online]. Available: https://hal.archives-ouvertes.fr/hal03343733/document

[22] M.-J. Youssef, J. Farah, C. A. Nour, and C. Douillard, "Waterfillingbased resource allocation techniques in downlink non-orthogonal multiple access (NOMA) with single-user MIMO," in IEEE Symposium on Computers and Communications (ISCC), 2017, pp. 499-506.

[23] R. Jain, D. Chiu, and W. Hawe, "A quantitative measure of fairness and discrimination for resource allocation in shared computer systems," DEC Technical Report 301, Sept. 1984.

[24] P. W. Wolniansky, G. J. Foschini, G. Golden, and R. A. Valenzuela, "V-BLAST: An architecture for realizing very high data rates over the rich-scattering wireless channel," in Signals, Systems, and Electronics, 1998. 